\title{
Production of purely gravitational dark matter
}

\author{
Yohei Ema, ${ }^{a, b}$ Kazunori Nakayama ${ }^{a, c}$ and Yong Tang ${ }^{a}$ \\ ${ }^{a}$ Department of Physics, Faculty of Science, The University of Tokyo, \\ Bunkyo-ku, Tokyo 113-0033, Japan \\ ${ }^{b}$ Theory Center, High Energy Accelerator Research Organization (KEK), \\ Tsukuba, Ibaraki 305-0801, Japan \\ ${ }^{c}$ Kavli IPMU (WPI), UTIAS, The University of Tokyo, \\ Kashiwa, Chiba 277-8583, Japan \\ E-mail: ema@post.kek.jp, kazunori@hep-th.phys.s.u-tokyo.ac.jp, \\ ytang@hep-th.phys.s.u-tokyo.ac.jp
}

ABSTRACT: In the purely gravitational dark matter scenario, the dark matter particle does not have any interaction except for gravitational one. We study the gravitational particle production of dark matter particle in such a minimal setup and show that correct amount of dark matter can be produced depending on the inflation model and the dark matter mass. In particular, we carefully evaluate the particle production rate from the transition epoch to the inflaton oscillation epoch in a realistic inflation model and point out that the gravitational particle production is efficient even if dark matter mass is much larger than the Hubble scale during inflation as long as it is smaller than the inflaton mass.

KeYwords: Classical Theories of Gravity, Cosmology of Theories beyond the SM

ARXIV EPRINT: 1804.07471 


\section{Contents}

1 Introduction 1

2 Scalar field in cosmological background 2

2.1 Model and equations of motion 2

2.2 Quantization and adiabatic vacuum 3

2.3 Energy and number density 5

3 Production of purely gravitational dark matter $\quad 6$

3.1 Analytic estimation 6

3.1.1 Conformal coupling $\quad 6$

3.1.2 Minimal coupling 9

$\begin{array}{ll}3.2 & \text { Numerical simulation in realistic inflation model } \\ \end{array}$

4 Discussion $\quad 13$

A Gravitational particle production in a toy expansion model with confor$\begin{array}{ll}\text { mal coupling } & 16\end{array}$

\section{Introduction}

There are many particle physics models to explain cosmological dark matter (DM). Most models introduce (small) DM interactions with some other fields to obtain the desired DM abundance. In this respect, the simplest possibility is that DM interacts with other sectors only through gravity. We call it as the purely gravitational DM (PGDM). Interestingly, even in the case where DM interacts only through gravity, there are several processes which contribute to the DM particle production.

The so-called gravitational particle production often refers to the particle creation due to the expansion of the Universe, or the time dependence of the cosmic scale factor $[1,2]$. The classic paper [3] studied the scalar particle production during the transition from inflation to the matter dominated (MD) or radiation dominated (RD) Universe and found that number density of $n_{\chi} \sim H_{\text {inf }}^{3}$ is produced, where $H_{\text {inf }}$ denotes the Hubble scale during inflation, if the scalar particle $\chi$ is effectively massless or $m_{\chi} \ll H_{\text {inf }}$ with $m_{\chi}$ being the mass of $\chi$. Refs. $[4,5]$ considered the gravitational particle creation at the end of inflation as a mechanism for the production of supermassive DM. More recently, refs. [6, 7] pointed out that gravitational particle production always happens during the inflaton oscillation era after inflation, since the cosmic scale factor is also oscillating. There it is found that the production is most efficient at early epoch and the produced number density is $n_{\chi} \sim H_{\text {inf }}^{3}$, similar to that found in ref. [3]. It becomes clear that this estimation applies to $m_{\chi}$ as large 
as the inflaton mass $m_{\phi}$, even if it is much larger than $H_{\text {inf }}$. It may be easily understood once one regards the gravitational particle production due to the oscillating background as the "gravitational annihilation" of inflaton. ${ }^{1}$

On the other hand, refs. [11-14] studied the production of PGDM through the annihilation of standard model particles in thermal bath with s-channel graviton exchange. It may also be regarded as a kind of gravitational particle creation. The efficiency of DM production is dominated at earlier epoch or high cosmic temperature. However, in the very early epoch the Universe is dominated by the (coherently oscillating) inflaton field, hence the DM production is expected to be dominated by the annihilation of inflaton rather than particles in thermal bath in most cases [13].

In this paper we revisit the gravitational particle production in a realistic situation. It is often misunderstood that the gravitational particle production is not efficient if $m_{\chi} \gg H_{\text {inf }}$. It is not always true, however. In many inflation models, there is a large hierarchy between the inflaton mass scale and Hubble scale: $m_{\phi} \gg H_{\text {inf }}$. Gravitational particle production is efficient even for $m_{\phi}>m_{\chi} \gg H_{\mathrm{inf}}$. This point is not stressed in literature except for a few works $[6,7]$. Therefore we want to study the gravitational production in a comprehensive manner.

This paper is organized as follows. In section 2 some basics of a scalar field in the expanding background are described and equations to estimate the gravitational particle production are derived. In section 3 we (semi)-analytically and numerically evaluate the gravitational particle production rate in a realistic inflationary cosmology. Section 4 is devoted to summary and discussion.

\section{Scalar field in cosmological background}

\subsection{Model and equations of motion}

Let us consider an action

$$
S=\int d^{4} x \sqrt{-g}\left(\frac{1}{2}\left(M_{P}^{2}-\xi \chi^{2}\right) R-\frac{1}{2} g^{\mu \nu} \partial_{\mu} \phi \partial_{\nu} \phi-V(\phi)-\frac{1}{2} g^{\mu \nu} \partial_{\mu} \chi \partial_{\nu} \chi-\frac{1}{2} m_{\chi}^{2} \chi^{2}\right),
$$

where $M_{P}$ is the reduced Planck scale, $R$ is the Ricci scalar, $\phi$ denotes the inflaton field with $V(\phi)$ being its potential and $\chi$ denotes a real scalar field. It has a $Z_{2}$ symmetry under which $\chi$ changes its sign, and hence $\chi$ is stable and is a candidate of DM. We assume that $\chi$ does not have a direct coupling to the inflaton and other standard model fields. It interacts only through the metric or the gravity. The coupling strength to the gravity is controlled by the non-minimal coupling $\xi$. Pure Einstein gravity corresponds to $\xi=0$ and the conformal coupling corresponds to $\xi=1 / 6$.

We use the Friedmann-Robertson-Walker metric:

$$
g_{\mu \nu} d x^{\mu} d x^{\nu}=a^{2}(\tau)\left(-d \tau^{2}+d \vec{x}^{2}\right),
$$

\footnotetext{
${ }^{1}$ Refs. $[8,9]$ pointed out that there is a tachyonically resonant production if $\chi$ has a (large) non-minimal coupling to gravity. See also refs. [10].
} 
where $a(\tau)$ denotes the cosmic scale factor with $\tau$ being the conformal time, which is related to the physical time as $d t=a d \tau$. Defining $\tilde{\chi} \equiv a \chi$, the action of $\tilde{\chi}$ is given by

$$
S=\int d \tau d^{3} x \frac{1}{2}\left[\widetilde{\chi}^{\prime 2}-\left(\partial_{i} \widetilde{\chi}\right)^{2}-m_{\chi}^{(\mathrm{eff}) 2} \widetilde{\chi}^{2}\right], \quad m_{\chi}^{(\mathrm{eff}) 2} \equiv a^{2} m_{\chi}^{2}-(1-6 \xi) \frac{a^{\prime \prime}}{a},
$$

where the prime denotes the derivative with respect to $\tau$. Thus $\tilde{\chi}$ satisfies the equation of motion

$$
\widetilde{\chi}^{\prime \prime}-\partial_{i}^{2} \widetilde{\chi}+m_{\chi}^{(\mathrm{eff}) 2} \widetilde{\chi}=0
$$

Treated as classical background, $\phi$ has the following equation of motion,

$$
\ddot{\phi}+3 H \dot{\phi}+\frac{\partial V}{\partial \phi}=0
$$

where the dot denotes the derivative with respect to the physical time $t$ and the Hubble parameter $H$ is given by the Friedmann equation,

$$
H^{2}=\left(\frac{\dot{a}}{a}\right)^{2}=\frac{1}{3 M_{P}^{2}}\left(\frac{1}{2} \dot{\phi}^{2}+V(\phi)\right)
$$

with the $\chi$ contribution to the energy density neglected. Thus for any given inflation model, we can calculate the production rate of $\chi$ through the time dependence of the scale factor $a$ in (2.3). These equations are written in terms of the conformal time as

$$
\phi^{\prime \prime}+2 \mathcal{H} \phi^{\prime}+a^{2} \frac{\partial V}{\partial \phi}=0
$$

where the conformal Hubble parameter $\mathcal{H}$ is

$$
\mathcal{H}^{2} \equiv\left(\frac{a^{\prime}}{a}\right)^{2}=\frac{1}{3 M_{P}^{2}}\left(\frac{1}{2} \phi^{\prime 2}+a^{2} V\right) .
$$

The Friedmann equation of the second kind is given by

$$
\frac{a^{\prime \prime}}{a}=\frac{a^{2} R}{6}=\frac{4 a^{2} V-\phi^{2}}{6 M_{P}^{2}}
$$

\subsection{Quantization and adiabatic vacuum}

Since $m_{\chi}^{(\text {eff }) 2}$ is time-dependent in the expanding Universe, we should be careful about the choice of mode function and vacuum state. Let us define the Fourier mode as

$$
\widetilde{\chi}(\tau, \vec{x})=\int \frac{d^{3} k}{(2 \pi)^{3}}\left[a_{\vec{k}} \chi_{k}(\tau)+a_{-\vec{k}}^{\dagger} \chi_{k}^{*}(\tau)\right] e^{i \vec{k} \cdot \vec{x}},
$$

where $\chi_{k}=\chi_{-k}$ must be satisfied from the reality of $\chi$, and the ladder operator satisfies

$$
\left[a_{\vec{k}}, a_{\vec{k}^{\prime}}^{\dagger}\right]=(2 \pi)^{3} \delta\left(\vec{k}-\vec{k}^{\prime}\right), \quad\left[a_{\vec{k}}, a_{\vec{k}^{\prime}}\right]=\left[a_{\vec{k}}^{\dagger}, a_{\vec{k}^{\prime}}^{\dagger}\right]=0
$$


The Fourier mode satisfies the equation of motion:

$$
\chi_{k}^{\prime \prime}+\omega_{k}^{2} \chi_{k}=0, \quad \omega_{k}^{2} \equiv k^{2}+m_{\chi}^{(\mathrm{eff}) 2} .
$$

From the canonical commutation relation

$$
\left[\widetilde{\chi}(\vec{x}), \widetilde{\chi}^{\prime}\left(\vec{x}^{\prime}\right)\right]=i \delta\left(\vec{x}-\vec{x}^{\prime}\right)
$$

we obtain the normalization condition

$$
\chi_{k} \chi_{k}^{\prime *}-\chi_{k}^{*} \chi_{k}^{\prime}=i
$$

The vacuum state $|0\rangle$ is defined as $a_{\vec{k}}|0\rangle=0$ for some mode function $\chi_{k}$ at some initial time $\tau=\tau_{i}$. In the Heisenberg picture, the state does not evolve once we fix it at the initial time. Instead, the mode function develops with time, which may be interpreted as the particle production as will be shown later.

Given initial conditions, one can directly solve (2.12), but here we use a different technique [18]. Let us rewrite $\chi_{k}(\tau)$ as follows:

$$
\chi_{k}(\tau)=\alpha_{k}(\tau) v_{k}(\tau)+\beta_{k}(\tau) v_{k}^{*}(\tau), \quad v_{k}(\tau) \equiv \frac{1}{\sqrt{2 \omega_{k}}} \exp \left(-i \int \omega_{k} d \tau\right) .
$$

Since both $\alpha_{k}(\tau)$ and $\beta_{k}(\tau)$ are time-dependent, one can always write $\chi_{k}$ in this form. Still there is a degree of freedom for the choice of $\alpha_{k}(\tau)$ and $\beta_{k}(\tau)$. One can impose the following condition, which is consistent with the equation of motion (2.12):

$$
\alpha_{k}^{\prime} v_{k}=\frac{\omega_{k}^{\prime}}{2 \omega_{k}} v_{k}^{*} \beta_{k}, \quad \beta_{k}^{\prime} v_{k}^{*}=\frac{\omega_{k}^{\prime}}{2 \omega_{k}} v_{k} \alpha_{k}
$$

Instead of solving (2.12), one may solve (2.16), which is often much easier for the purpose of evaluating the particle production numerically. Note that (2.14) ensures

$$
\left|\alpha_{k}(\tau)\right|^{2}-\left|\beta_{k}(\tau)\right|^{2}=1
$$

In order to extract the number density from $\alpha_{k}$ and $\beta_{k}$, we must first specify the initial condition for the mode function (which corresponds to the choice of the vacuum state) and the observer state which counts the number density. In general, there is no preferred choice for the states of the vacuum and the observer in curved spacetime. In our case, however, we may formally assume that the spacetime is asymptotically static in the far past $\tau \rightarrow-\infty$ (deep in the inflationary era) as well as in the far future $\tau \rightarrow+\infty$ (deep in the MD or RD era). In such a case, it is natural to take the vacuum/observer as the negative frequency modes in the limit $\tau \rightarrow-\infty / \infty$, respectively. The negative frequency mode approaches to

$$
\chi_{k}(\tau) \rightarrow \frac{1}{\sqrt{2 k}} e^{-i k \tau}
$$

in the limit $\tau \rightarrow-\infty$. Thus we take $\alpha\left(\tau_{i}\right)=1$ and $\beta\left(\tau_{i}\right)=0$ in the limit $\tau_{i} \rightarrow-\infty$ as the initial condition. Note that it represents the adiabatic vacuum of the infinite order [2], 
since the spacetime is assumed to be static in the far past/future regions. ${ }^{2}$ In our numerical calculation, however, it is of course impossible to run from $\tau_{i}=-\infty$, and hence we start our numerical calculation with $\alpha\left(\tau_{i}\right)=1$ and $\beta\left(\tau_{i}\right)=0$ at some large but finite $\tau_{i}$. We will discuss how to infer the result with $\tau_{i} \rightarrow-\infty$ from our numerical results with finite $\tau_{i}$ in the next section.

Here is a comment on the size of $m_{\chi}^{2}$ and $\xi$. The exact solution to (2.12) during the (pure) de Sitter era with the initial condition (2.18) is given by

$$
\chi_{k}(\tau)=e^{\frac{i(2 \nu+1) \pi}{4}} \frac{1}{\sqrt{2 k}} \sqrt{\frac{-\pi k \tau}{2}} H_{\nu}^{(1)}(-k \tau), \quad \nu^{2} \equiv \frac{9}{4}-12 \xi-\frac{m_{\chi}^{2}}{H^{2}},
$$

where $H_{\nu}^{(1)}$ denotes the Hankel function of the first kind. In this paper we mainly concentrate on the case of $\nu^{2}<1 / 4$. Otherwise long wave superhorizon fluctuation develops and $\chi$ obtains a homogeneous classical field value ${ }^{3}$ and we must take account of the coherent oscillation of the homogeneous $\chi$ field for estimation of the final DM abundance. For the small nonminimal coupling $|\xi| \leq 1 / 6, \nu^{2}<1 / 4$ roughly means that $\chi$ is heavier than the Hubble scale during and after inflation. For the nonminimal coupling $\xi \geq 1 / 6, \nu^{2}<1 / 4$ always holds even if $m_{\chi}^{2} \ll H^{2}$ as far as $m_{\chi}^{2}$ is positive.

\section{$2.3 \quad$ Energy and number density}

In order to evaluate the amount of created particles in the cosmological background, one must define the energy or number density. Of course, they are actually UV divergent and hence we need to carefully renormalize them. The energy momentum tensor of $\chi$ is given by $\sqrt{-g} T_{\mu \nu}^{\chi}=-2 \delta\left(\sqrt{-g} \mathcal{L}_{\chi}\right) / \delta g^{\mu \nu}[2]$ :

$$
\begin{aligned}
T_{\mu \nu}^{\chi}= & (1-2 \xi) \partial_{\mu} \chi \partial_{\nu} \chi+\left(2 \xi-\frac{1}{2}\right) g_{\mu \nu}(\partial \chi)^{2}+2 \xi\left(g_{\mu \nu} \chi \square \chi-\chi \nabla_{\mu} \partial_{\nu} \chi\right) \\
& +\xi G_{\mu \nu} \chi^{2}-\frac{1}{2} m_{\chi}^{2} g_{\mu \nu} \chi^{2}
\end{aligned}
$$

where $G_{\mu \nu}$ denotes the Einstein tensor and partial derivative is taken with respect to physical time $t$. The energy density may be defined through $\rho_{\chi}=\left\langle 0\left|T_{00}^{\chi}\right| 0\right\rangle$. Using (2.10), we obtain

$$
\begin{aligned}
& a^{4} \rho_{\chi}=\int \frac{d^{3} k}{(2 \pi)^{3}} \frac{1}{2}\left[\left|\chi_{k}^{\prime}\right|^{2}+\left(k^{2}+a^{2} m_{\chi}^{2}\right)\left|\chi_{k}\right|^{2}+(1-6 \xi)\left\{\mathcal{H}^{2}\left|\chi_{k}\right|^{2}-\mathcal{H}\left(\chi_{k} \chi_{k}^{* \prime}+\chi_{k}^{*} \chi_{k}^{\prime}\right)\right\}\right] \\
& =\int \frac{d^{3} k}{(2 \pi)^{3}} \frac{\omega_{k}}{2}\left[\left(1+2\left|\beta_{k}\right|^{2}\right)\left(1+(1-6 \xi) \frac{2 \mathcal{H}^{2}+\mathcal{H}^{\prime}}{2 \omega_{k}^{2}}\right)+(1-6 \xi)\left(\frac{2 i \mathcal{H} \omega_{k}+\mathcal{H}^{\prime}+\mathcal{H}^{2}}{\omega_{k}} \alpha_{k} \beta_{k}^{*} v_{k}^{2}+\text { h.c. }\right)\right] .
\end{aligned}
$$

\footnotetext{
${ }^{2}$ One can show that our definition of $f_{\chi}$ is equivalent to the number density defined by the Bogoliubov coefficient where the vacuum and the observer states are taken as the zero-th order adiabatic vacuum as long as $\omega_{k}^{\prime} / \omega_{k}=0$ at $\tau=\tau_{i}$ and $\tau_{f}$, where $\tau_{f}$ is the conformal time at which the number density is evaluated. Since the adiabatic expansion is exact in the limit in the far past/future regions, our $f_{\chi}$ coincides with the number density defined by the adiabatic vacuum of the infinite order for $-\tau_{i}, \tau_{f} \rightarrow \infty$ as well.

${ }^{3}$ The long wavelength limit is $H_{\nu}^{(1)}(-k \tau) \rightarrow-(i / \pi) \Gamma(\nu)(-k \tau / 2)^{-\nu}$ for $k \tau \rightarrow 0$. Note that $m_{\chi}^{(\text {eff }) 2}>0$ always holds if $\nu^{2}<1 / 4$ during inflation and hence $\omega_{k}$ is real for all the wavenumber $k$.
} 
If we naively introduce a physical UV cutoff scale $\Lambda$, we find terms like $\rho_{\chi} \propto \Lambda^{4}, H^{2} \Lambda^{2}$ and $H^{4} \log \Lambda$. These divergences are renormalized by the constant term, Planck constant and the coefficient of the $R^{2}$ term in the action, respectively [2]. ${ }^{4}$ Since we want to calculate the energy density of $\chi$ in the far future in which $\mathcal{H} \rightarrow 0$ and $\mathcal{H}^{\prime} \rightarrow 0$, one can safely define the renormalized energy density as

$$
a^{4}(\tau) \rho_{\chi}^{(\text {ren })}(\tau)=\int \frac{d^{3} k}{(2 \pi)^{3}} \omega_{k} f_{\chi}(k, \tau), \quad f_{\chi}(k, \tau) \equiv|\beta(k, \tau)|^{2},
$$

where $f_{\chi}(k)$ denotes the phase space density of the $\chi$ particle with physical momentum $k / a$. Similarly, the number density is given by

$$
a^{3}(\tau) n_{\chi}(\tau)=\int \frac{d^{3} k}{(2 \pi)^{3}} f_{\chi}(k, \tau) .
$$

Therefore, given an cosmological model, we can calculate the evolution $\beta_{k}$ by using (2.16) and then estimate the number of produced particles. In particular, in the most cases of our interest $\beta_{k}$ is so small that we can safely take $\alpha_{k} \simeq 1$. Then we have

$$
\beta_{k}\left(\tau_{f}\right) \simeq \int_{\tau_{i}}^{\tau_{f}} \frac{\omega_{k}^{\prime}}{2 \omega_{k}} e^{-2 i \Omega_{k}(\tau)} d \tau, \quad \Omega_{k}(\tau) \equiv \int_{\tau_{i}}^{\tau} \omega_{k} d \tau
$$

As we discussed in the previous subsection, we take the limit $\tau_{i} \rightarrow-\infty$, and evaluate $f_{\chi}$ at $\tau_{f} \rightarrow \infty$ where (2.23) is rigorous, assuming that the spacetime is static in these regions.

\section{Production of purely gravitational dark matter}

\subsection{Analytic estimation}

\subsubsection{Conformal coupling}

Now let us evaluate $\beta_{k}$ through (2.25). In the following we always assume $m_{\chi} \lesssim m_{\phi}$. First, let us consider the case of conformal coupling $\xi=1 / 6$ for simplicity. Of course, in the massless limit $m_{\chi}=0$ the $\chi$ field does not feel the Universe expanding due to the conformality and no particle production occurs. However, for finite $m_{\chi}$, particle production happens. We can approximate $\Omega_{k}$ as

$$
\Omega_{k}(\tau) \simeq\left\{\begin{array}{ll}
k \tau & \text { for } \quad k^{2} \gg a^{2} m_{\chi}^{2} \\
m_{\chi} t & \text { for } \quad k^{2} \ll a^{2} m_{\chi}^{2}
\end{array},\right.
$$

where we have ignored additional irrelevant constant. The integrand is given by

$$
\frac{\omega_{k}^{\prime}}{2 \omega_{k}}=\frac{\mathcal{H}}{2} \frac{a^{2} m_{\chi}^{2}}{k^{2}+a^{2} m_{\chi}^{2}} \simeq\left\{\begin{array}{lll}
\frac{\mathcal{H} a^{2} m_{\chi}^{2}}{2 k^{2}} & \text { for } & k^{2} \gg a^{2} m_{\chi}^{2} \\
\frac{\mathcal{H}}{2} & \text { for } & k^{2} \ll a^{2} m_{\chi}^{2}
\end{array} .\right.
$$

\footnotetext{
${ }^{4}$ As for the quadratic divergence, one can explicitly check that a term like $\dot{H} \Lambda^{2}$ is cancelled out.
} 
The integrand approaches to zero both in the limit $\tau \rightarrow-\infty$ (deep in the inflationary era) and $\tau \rightarrow+\infty$ (deep in the MD or RD era). Thus the integration (2.25) is well-defined,

$$
\beta_{k}\left(\tau_{f}\right) \simeq \int_{\tau_{i}}^{\tau_{k}} \frac{\mathcal{H} a^{2} m_{\chi}^{2}}{2 k^{2}} e^{-2 i k \tau} d \tau+\int_{t_{k}}^{t_{f}} \frac{H}{2} e^{-2 i m_{\chi} t} d t,
$$

where $\tau_{k}$ is defined as the conformal time at which $a\left(\tau_{k}\right) m_{\chi}=k$. Now we need the time dependence of the scale factor $a(t)$ for evaluating this integral. As explicitly noticed in ref. [6], the scale factor $a(t)$ contains rapidly oscillating part during the inflaton coherent oscillation, as

$$
a(t) \simeq\langle a(t)\rangle\left(1-\frac{\left(\phi-v_{\phi}\right)^{2}}{8 M_{P}^{2}}\right)
$$

where $v_{\phi}$ is the final VEV of inflaton and $\langle a(t)\rangle$ is the time-averaged value over the inflaton oscillation period. The particle production may be caused by both the time-dependence of the averaged value $\langle a(t)\rangle$ and also by the rapidly oscillating part. We stress that such a clear separation is possible only in the harmonic inflaton oscillation regime and distinction between "slowly changing" part and "rapidly changing" part is not well defined in general. Nevertheless, this picture helps the analytic understandings of the particle production.

First let us focus on the effect by the time-averaged part $\langle a(t)\rangle$. We discuss this contribution in detail in appendix $\mathrm{A}$ and the resultant phase space distribution is given by (A.2) and (A.3):

$$
f_{\chi}(k) \sim\left\{\begin{array}{ll}
\exp \left(-\frac{c m_{\chi}}{H_{\mathrm{inf}}}\right) & \text { for } k<a_{\mathrm{end}} m_{\chi} \\
\frac{a_{\mathrm{end}}^{4} m_{\chi}^{4}}{k^{4}} \exp \left(-\frac{c k}{a_{\mathrm{end}} H_{\mathrm{inf}}}\right)+\exp \left[-c\left(\frac{k}{k_{c}}\right)^{3 / 2}\right] & \text { for } k>a_{\text {end }} m_{\chi}
\end{array},\right.
$$

where $c \sim 5$ and $a_{\text {end }}$ denotes the scale factor at the end of inflation and $k_{c}$ is defined by the momentum so that $m_{\chi} t_{k}=1$.

Next we consider the effect from the rapidly oscillating part in (3.4). The particle production due to such a rapidly oscillating effective mass term is analogous to the process known as the narrow resonance due to the oscillating scalar field [15-18]. In our situation the parametric resonance effect is neglected since the Hubble expansion is fast enough to expel the created particles from the resonance band in the momentum space [6] and in such a case the production rate is the same as the perturbative decay/annihilation rate. The effective inflaton annihilation rate in this case is

$$
\Gamma_{\phi \phi \rightarrow \chi \chi} \simeq \frac{C}{16 \pi} \frac{\Phi^{2}}{M_{P}^{4}} \frac{m_{\chi}^{4}}{m_{\phi}},
$$

if $m_{\chi} \lesssim m_{\phi}$, where $\Phi$ denotes the oscillation amplitude of the inflaton field and $C$ is a numerical factor. Thus the created number density during one Hubble time is given by

$$
n_{\chi} \simeq \frac{9 C}{4 \pi}\left(\frac{m_{\chi}}{m_{\phi}}\right)^{4} H^{3} .
$$


One immediately notices that the dominant contribution comes from the earliest epoch,

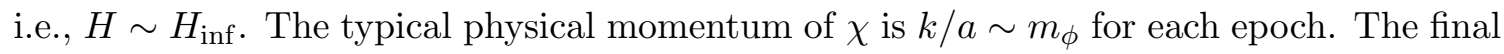
momentum distribution of $\chi$ is not exponentially suppressed for large $k$ since the production continues until the inflaton decays. We obtain the following approximated form:

$$
f_{\chi}(k, \tau) \sim \begin{cases}\left(\frac{H_{\mathrm{inf}}}{m_{\phi}}\right)^{3}\left(\frac{m_{\chi}}{m_{\phi}}\right)^{4} & \text { for } \quad k<a_{\mathrm{end}} m_{\phi}, \\ \left(\frac{H_{\mathrm{inf}}}{m_{\phi}}\right)^{3}\left(\frac{m_{\chi}}{m_{\phi}}\right)^{4}\left(\frac{k}{a_{\mathrm{end}} m_{\phi}}\right)^{-9 / 2} & \text { for } \quad k>a_{\mathrm{end}} m_{\phi},\end{cases}
$$

and there will be an exponential cutoff at $k \sim a\left(t=\Gamma_{\text {inf }}^{-1}\right) m_{\phi}$ where $\Gamma_{\text {inf }}$ denotes the total decay width of the inflaton. ${ }^{5}$ This contribution is not suppressed even for $m_{\chi} \gg H_{\text {inf }}$. Note also that the low momentum behavior of (3.8) may not be so simple because of the nontrivial time scale of the inflaton dynamics during the transition from inflation to the reheating era. The final momentum distribution is roughly the sum of (3.8) and (3.5). In realistic situation, as we will see later, these two contribution should be smoothly connected because the "end" of inflation is rather vague and the inflaton oscillation is far from harmonic at the early stage of the reheating and its typical time scale changes from $H_{\text {inf }}$ to $m_{\phi}$ gradually. The number density is then given by

$$
n_{\chi}(\tau) \sim H_{\mathrm{inf}}^{3}\left(\frac{a_{\mathrm{end}}}{a(\tau)}\right)^{3}\left[\mathcal{C}\left(\frac{m_{\chi}}{m_{\phi}}\right)^{4}+\eta \frac{m_{\chi}}{H_{\mathrm{inf}}}\right]
$$

where we will numerically find $\mathcal{C} \sim 10^{-3}-10^{-2}$ and

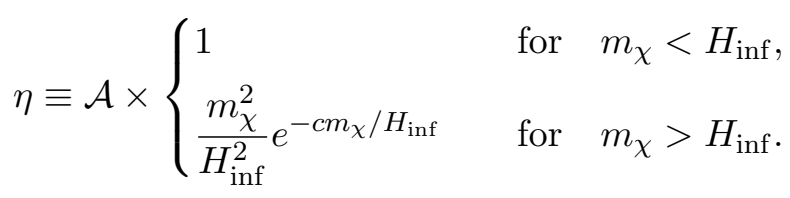

with $\mathcal{A} \sim 6 \times 10^{-4}$ for $m_{\chi} \lesssim H_{\text {inf }}$. Thus the contribution from the pure Hubble expansion likely to dominate for $m_{\chi}<H_{\text {inf }}$ while that from the oscillation effect (3.8) becomes important for $m_{\chi}>H_{\text {inf }}$. In the latter case, we can evaluate the present DM energy density from the gravitational production divided by the entropy density as

$$
\begin{aligned}
\frac{\rho_{\chi}^{(\mathrm{GP})}}{s} & \sim \frac{\mathcal{C}}{4} \frac{m_{\chi} H_{\mathrm{inf}} T_{\mathrm{R}}}{M_{P}^{2}}\left(\frac{m_{\chi}}{m_{\phi}}\right)^{4} \\
& \simeq 4 \times 10^{-10} \mathrm{GeVC}\left(\frac{m_{\chi}}{10^{9} \mathrm{GeV}}\right)\left(\frac{H_{\mathrm{inf}}}{10^{9} \mathrm{GeV}}\right)\left(\frac{T_{\mathrm{R}}}{10^{10} \mathrm{GeV}}\right)\left(\frac{m_{\chi}}{m_{\phi}}\right)^{4}
\end{aligned}
$$

for $m_{\chi}<m_{\phi}$, where $T_{\mathrm{R}}$ denotes the reheating temperature after inflation and we assumed that the inflaton coherent oscillation behaves as non-relativistic matter.

\footnotetext{
${ }^{5}$ For the completion of the reheating, we need to introduce inflaton coupling to some other sector.
} 


\subsubsection{Minimal coupling}

Now let us consider the case of minimal coupling, $\xi=0$. In this case, we have

$$
m_{\chi}^{(\mathrm{eff}) 2}=a^{2}\left(m_{\chi}^{2}-\frac{R}{6}\right)
$$

and we obtain

$$
\frac{\omega_{k}^{\prime}}{2 \omega_{k}} \simeq \frac{1}{2} \frac{\mathcal{H} a^{2} m_{\chi}^{2}-\left(a^{2} R\right)^{\prime} / 12}{k^{2}+a^{2} m_{\chi}^{2}}
$$

where we have assumed $m_{\chi} \gtrsim H_{\text {inf }}$ to avoid the growth superhorizon modes as already mentioned. The first term is the same as the conformal coupling case and hence we focus on the second term hereafter. In the following we also assume $m_{\chi} \lesssim m_{\phi}$.

The Ricci scalar $R$ is expressed in terms of $\phi$ as (2.9) and hence it is a rapidly oscillating function when the inflaton oscillates coherently. Therefore we can divide $R$ into its oscillation averaged part and the oscillating part, schematically as $R \sim\langle R\rangle+m_{\phi}^{2}\left(\phi-v_{\phi}\right)^{2} / M_{P}^{2}$. First let us consider the effect due to the averaged part. After the partial integration, the integral (2.25) is expressed as

$$
\beta_{k} \sim \frac{-i}{12} \int \frac{a^{2}\langle R\rangle}{\omega_{k}} e^{-2 i \Omega_{k}} d \tau
$$

It may be dominated around the transition $\tau \sim \tau_{\text {end }}$ where $a^{2} R / \omega_{k}$ takes its maximum value. Thus we roughly have ${ }^{6}$

$$
f_{\chi}(k) \sim\left\{\begin{array}{ll}
\frac{H_{\mathrm{inf}}^{2}}{m_{\chi}^{2}} \exp \left(-\frac{c m_{\chi}}{H_{\mathrm{inf}}}\right) & \text { for } k<a_{\mathrm{end}} m_{\chi} \\
\frac{a_{\mathrm{end}}^{2} H_{\mathrm{inf}}^{2}}{k^{2}} \exp \left(-\frac{c k}{a_{\mathrm{end}} H_{\mathrm{inf}}}\right) & \text { for } k>a_{\mathrm{end}} m_{\chi}
\end{array} .\right.
$$

Next we consider a particle production due to the oscillating part in $R$. As already mentioned in the case of conformal coupling, such a rapidly oscillating term induces particle production similar to the standard narrow resonance. On these grounds, the effective inflaton annihilation rate during the inflaton coherent oscillation is given by

$$
\Gamma_{\phi \phi \rightarrow \chi \chi} \simeq \frac{C}{16 \pi} \frac{\Phi^{2}}{M_{P}^{4}} m_{\phi}^{3}
$$

if $m_{\chi} \lesssim m_{\phi}$ where $C$ is a numerical factor and $\Phi$ denotes the inflaton oscillation amplitude. Thus the created number density during one Hubble time is given by

$$
n_{\chi} \simeq \frac{9 C}{4 \pi} H^{3} .
$$

\footnotetext{
${ }^{6}$ Even for $m_{\chi} \ll H_{\text {inf }}$, the result for the high momentum mode $k>a_{\text {end }} H_{\text {inf }}$ looks similar to that for the high momentum limit of the case of $m_{\chi} \gtrsim H_{\text {inf }}$. These high momentum contribution goes as $n_{\chi} \sim H_{\text {inf }}^{3}\left(a_{\text {end }} / a(t)\right)^{3}$, as given in ref. [3], but the long wavelength contribution can be dominant in such a case. See section 4 .
} 
Again, the dominant contribution comes from the earliest epoch, i.e., $H \sim H_{\text {inf }}$. Similarly to the case studied in the conformal coupling case, the typical physical momentum of $\chi$ is $k / a \sim m_{\phi}$ for each epoch and the final momentum distribution of $\chi$ is not exponentially suppressed for large $k$ since the production continues until the inflaton decays. We expect that the momentum distribution looks like

$$
f_{\chi}(k, \tau) \sim \begin{cases}\left(\frac{H_{\mathrm{inf}}}{m_{\phi}}\right)^{3} & \text { for } k<a_{\mathrm{end}} m_{\phi}, \\ \left(\frac{H_{\mathrm{inf}}}{m_{\phi}}\right)^{3}\left(\frac{k}{a_{\mathrm{end}} m_{\phi}}\right)^{-9 / 2} & \text { for } k>a_{\mathrm{end}} m_{\phi},\end{cases}
$$

and there is an exponential cutoff at $k \sim a\left(t=\Gamma_{\text {inf }}^{-1}\right) m_{\phi}$. It is not suppressed even for $m_{\chi} \gg H_{\text {inf }}$. Note again that the low momentum behavior of (3.18) may not be so simple because of the nontrivial time scale of the inflaton dynamics during the transition from inflation to the reheating era. As mentioned above, there are another contribution as (3.15), (3.8) and (3.5). Again we stress that, in realistic situation, these contributions should be smoothly connected because we cannot strictly define the "end" of inflation and the typical time scale of the inflaton motion changes from $H_{\text {inf }}$ to $m_{\phi}$ gradually around the transition epoch. The number density is dominated by that from the oscillation effect (3.18) for $m_{\chi} \gg H_{\text {inf }}$ and two contributions are comparable for $m_{\chi} \lesssim H_{\text {inf }}$. In any case, the number density is given by

$$
n_{\chi}(\tau) \sim \mathcal{C} H_{\text {inf }}^{3}\left(\frac{a_{\text {end }}}{a(\tau)}\right)^{3},
$$

for $H_{\text {inf }}<m_{\chi}<m_{\phi}$ where we numerically find $\mathcal{C} \sim 10^{-2}$. The present DM energy density from gravitational production divided by the entropy density is then given by

$$
\frac{\rho_{\chi}^{(\mathrm{GP})}}{s} \sim \frac{\mathcal{C}}{4} \frac{m_{\chi} H_{\mathrm{inf}} T_{\mathrm{R}}}{M_{P}^{2}} \simeq 3 \times 10^{-10} \mathrm{GeVC}\left(\frac{m_{\chi}}{10^{9} \mathrm{GeV}}\right)\left(\frac{H_{\mathrm{inf}}}{10^{9} \mathrm{GeV}}\right)\left(\frac{T_{\mathrm{R}}}{10^{10} \mathrm{GeV}}\right),
$$

for $m_{\chi}<m_{\phi}$ and $T_{\mathrm{R}}$ denotes the reheating temperature after inflation and we assumed that the inflaton coherent oscillation behaves as non-relativistic matter.

\subsection{Numerical simulation in realistic inflation model}

Let us now calculate the phase space density numerically. As a concrete inflation model, let us consider the hilltop inflation or new inflation models [19-25]. The inflaton potential is given by

$$
V(\phi)=M^{4}\left[1-\left(\frac{\phi}{v_{\phi}}\right)^{n}\right]^{2}
$$

where $n \geq 6$ is favored from the recent cosmological data [26]. In a numerical calculation we take $n=6$. This model is of our interest because it in general predicts a large hierarchy between $m_{\phi}$ and $H_{\text {inf }}$. Actually we have $m_{\phi}=\sqrt{2} n M^{2} / v_{\phi} \gg H_{\text {inf }} \simeq M^{2} /\left(\sqrt{3} M_{P}\right)$ for $v_{\phi} \ll M_{P}$. The field value at the end of inflation is defined by

$$
\phi_{\text {end }}=v_{\phi}\left(\frac{v_{\phi}}{\sqrt{2} n M_{P}}\right)^{1 /(n-1)}
$$



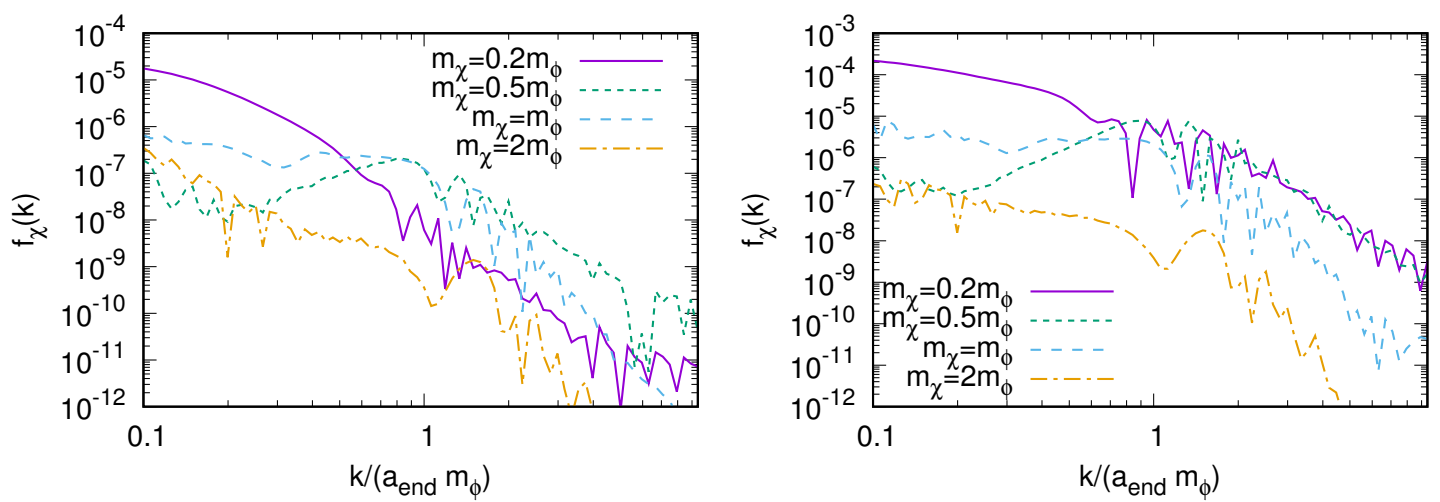

Figure 1. The phase space density of $\chi$ after the gravitational particle production in hilltop inflation model with $v_{\phi}=0.5 M_{P}$. (Left) The case of conformal coupling $\xi=1 / 6$. We have taken $m_{\chi}=(0.2,0.5,1,2) \times m_{\phi}$ for each line. (Right) The same as the left but for the minimal coupling $\xi=0$.

where the slow-roll parameter $\epsilon \equiv\left(M_{P} \partial V / \partial \phi\right)^{2} /(\sqrt{2} V)^{2}$ becomes equal to unity. The dimensionless power spectrum of the large scale curvature perturbation is given by

$$
\mathcal{P}_{\zeta} \simeq \frac{\left[2 n\left((n-2) N_{e}\right)^{n-1}\right]^{2 /(n-2)}}{12 \pi^{2}} \frac{M^{4}}{\left(v_{\phi}^{n} M_{P}^{n-4}\right)^{2 /(n-2)}},
$$

where $N_{e} \simeq 50-60$ denotes the e-folding number at which the perturbation with the present horizon scale exits the horizon. The Planck observation suggests $\mathcal{P}_{\zeta} \simeq 2.2 \times 10^{-9}$ [28].

We numerically solved (2.16) along with the background (2.7) and (2.8). Figure 1 shows the resulting phase space distribution $f_{\chi}(k)$ for the conformal coupling case $\xi=1 / 6$ (left) and the minimal coupling case $\xi=0$ (right). We have taken $v_{\phi}=0.5 M_{P}$, which means $m_{\phi} / H_{\mathrm{inf}} \simeq 29$, and $M$ is taken to satisfy the observed density perturbation. Three lines correspond to $m_{\chi}=(0.2,0.5,1,2) \times m_{\phi}$, respectively. The initial condition is set to be $\phi\left(\tau_{i}\right) \simeq 0.4 \phi_{\text {end }}$ with $\tau_{i}=-1 /\left(a\left(\tau_{i}\right) H_{\text {inf }}\right)$ where $a\left(\tau_{i}\right)$ can be arbitrary value. The final evaluation time is taken to be $\tau_{f}=-\tau_{i}$. The wave number in the horizontal axis is normalized by $a_{\text {end }} m_{\phi}$ where $a_{\text {end }}$ denotes the scale factor at the end of slow-roll regime $\phi=\phi_{\text {end }}$. The results are consistent with (3.8) for the conformal coupling case $\xi=1 / 6$ and (3.18) for the minimal coupling $\xi=0$ for the high momentum mode $k \gtrsim a_{\text {end }} m_{\phi}$. In particular, the overall size of $f_{\chi}$ is almost the same as long as $m_{\phi} \gtrsim m_{\chi}$ for $\xi=0$, and hence we conclude that the particles with $m_{\phi} \gtrsim m_{\chi}$ are indeed produced even if $m_{\chi} \gg H_{\text {inf }}$. On the other hand, the overall size of $f_{\chi}$ decreases as $m_{\chi}$ increases beyond $m_{\phi}$ even for $\xi=0$ (see the $m_{\chi}=2 m_{\phi}$ line in the right panel). The lower momentum behavior comes mainly from the transition epoch from inflation to the oscillation epoch, which is difficult to discuss analytically, but the estimations (3.11) and (3.20) remain valid because the number or energy density is dominated by the mode around $k \sim a_{\text {end }} m_{\phi} \cdot{ }^{7}$

\footnotetext{
${ }^{7}$ If $v_{\phi}$ is much smaller than $M_{P}$, the coherent oscillation just after inflation is not harmonic and the oscillation period can be much longer than $m_{\phi}^{-1}$. Moreover, tachyonic instability makes the inflaton field
} 

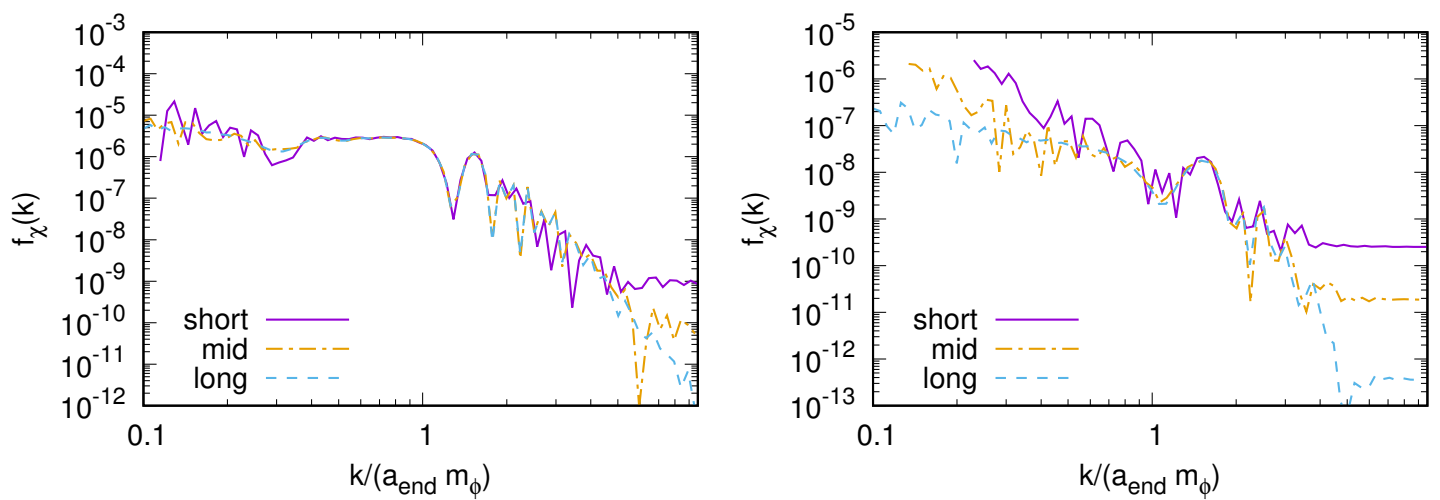

Figure 2. The phase space density of $\chi$ after the gravitational particle production for the minimal coupling $\xi=0$. The three lines "short", "mid" and "long" correspond to the duration of inflation in a numerical calculation. We have taken $m_{\chi}=m_{\phi}$ (left) and $m_{\chi}=2 m_{\phi}$ (right).

Now we comment on subtlety in evaluating $f_{\chi}(k)$. As we discussed in the previous section, we formally define the particle number density by taking $-\tau_{i}, \tau_{f} \rightarrow \infty$. In our numerical calculation, however, we take some finite $\tau_{i}$ and $\tau_{f}$ due to the limitation of computational cost, and hence we should carefully subtract effects of finite $\tau_{i}$ and $\tau_{f}$. More precisely, we want to take the limit $-k \tau_{i}, k \tau_{f} \rightarrow \infty$, since $\tau_{i}$ itself can be taken arbitrarily. For fixed physical momentum $k / a_{\mathrm{end}}$, such a limit can be achieved by taking the duration of inflation long in a numerical calculation. In order to identify such effects, we plot $f_{\chi}(k)$ for different initial condition in figure 2. Three lines correspond to different initial condition, $\phi_{i}=(0.38,0.41,0.44) \times \phi_{\text {end }}$ for "long," "mid" and "short," respectively. The DM mass is taken to be $m_{\chi}=m_{\phi}$ (left) and $m_{\chi}=2 m_{\phi}$ (right). The wave number in the horizontal axis is normalized by $a_{\text {end }} m_{\phi}$. As seen from the left panel, the flat part at large $k$ is initial condition dependent and becomes smaller as the initial time is taken to be earlier. The following simple example may be helpful to understand this behavior. Let us consider the integral:

$$
I(k)=\int_{\tau_{i}}^{\tau_{f}} \frac{e^{i k \tau}}{\tau^{2}+\tau_{0}^{2}} d \tau
$$

where $\tau_{0}$ is a real number. In the limit $-\tau_{i}=\tau_{f}=\infty$, we can exactly solve it by using the residue theorem and obtain exponential form: $I(k)=\pi e^{-k \tau_{0}} / \tau_{0}$. If we take large but finite $-\tau_{i}=\tau_{f}\left(\gg \tau_{0}\right)$, we instead have power law tail as $I(k) \sim \pi e^{-k \tau_{0}} / \tau_{0}+1 /\left(\tau_{f}^{2} k\right)$. The integrand is more complicated in a realistic situation, but we expect that a similar phenomena occur in our numerical calculation. Therefore the flat part at large $k$ is interpreted as an effect of finite $\tau_{i}$ and $\tau_{f}$, and we expect that it disappears for $-\tau_{i}, \tau_{f} \rightarrow-\infty$. On the other hand, the modes with smaller $k$ are not affected by the change of the initial condition, and hence are expected to be intact in the limit $-\tau_{i}, \tau_{f} \rightarrow \infty$. We have checked that the result in figure 1 is not affected by the change of the initial condition, and hence we expect that it

inhomogeneous [27]. These facts make robust calculation extremely difficult, although still the inflaton remains non-relativistic and we expect that the estimation here makes sense. 
provides good estimation of $f_{\chi}$ in the limit $-\tau_{i}, \tau_{f} \rightarrow \infty$. This issue is related to the choice of the initial condition at $\tau=\tau_{i}$, as discussed in the context of Bunch-Davies vacuum to some (or infinite) adiabatic order [2]. If we could carefully choose the initial conditions of $\alpha_{k}$ and $\beta_{k}$ at $\tau=\tau_{i}$ so that they match the solution with $\alpha_{k}=1$ and $\beta_{k}=0$ at $\tau=-\infty$, the $\tau_{i}$ dependence would be gone.

It is worth noting here that we do not actually know the initial condition of the Universe. Inflation should last at least for $\sim 60$ e-foldings, but the dynamics before the "observable" inflation is unclear. Therefore, strictly speaking, taking the limit $\tau_{i} \rightarrow-\infty$ may not be justified in the real Universe. Similarly, the present Universe has of course finite age, and we cannot formally take $\tau_{f} \rightarrow \infty$. We expect that taking these limits will give good estimation practically, but it would be interesting that information of the initial condition of the Universe may be contained in the momentum distribution.

\section{Discussion}

We studied the gravitational production of scalar PGDM by tracing the evolution of its wave function from inflation to the reheating era. We confirmed that PGDM mass with $m_{\chi} \gg H_{\text {inf }}$ can be efficiently produced as long as $m_{\chi} \lesssim m_{\phi}$, where $H_{\text {inf }}$ and $m_{\phi}$ denote the Hubble scale during inflation and the inflaton mass, respectively. Roughly, PGDM number density of $\sim H_{\text {inf }}^{3}$ is produced even for $H_{\text {inf }} \ll m_{\chi}\left(\lesssim m_{\phi}\right)$ at the end of inflation and the beginning of the inflaton oscillation. However, we note that our results depend on the assumption that the inflaton field remains homogeneous over the horizon scale until the inflaton coherent oscillation becomes purely harmonic. In actual situation, there can be some instability that tend to make the inflaton field inhomogeneous. Although our results may not be affected much unless the inflaton field becomes highly relativistic, further investigation is needed in order to correctly estimate the PGDM abundance in various inflation models.

So far we have focused on the case of $m_{\chi} \gtrsim H_{\text {inf }}$ in order to avoid the growth of large scale fluctuation during inflation. If $m_{\chi} \ll H_{\text {inf }}$, the superhorizon modes of the $\chi$ field are generated during inflation, ${ }^{8}$ and it will be effectively regarded as the homogeneous mode, which results in the coherent oscillation of $\chi$ field after inflation. This is another source of the DM production. Typical field variance averaged over the superhorizon mode is derived from the solution $(2.19)$ as $[29,30]$

$$
\left\langle\chi^{2}\right\rangle \simeq \frac{3 H_{\mathrm{inf}}^{4}}{8 \pi^{2} m_{\chi}^{2}}
$$

In a horizon patch, the $\chi$ field is almost homogeneous with its typical value given by $\sqrt{\left\langle\chi^{2}\right\rangle}$. The $\chi$ field then begins a coherent oscillation when $H \sim m_{\chi}$. The abundance of the coherent oscillation is estimated as

$$
\frac{\rho_{\chi}^{(\mathrm{CO})}}{s} \simeq \frac{T_{\mathrm{R}}}{8} \frac{\left\langle\chi^{2}\right\rangle}{M_{P}^{2}} \simeq 8 \times 10^{-12} \mathrm{GeV}\left(\frac{H_{\mathrm{inf}}}{10^{9} \mathrm{GeV}}\right)^{4}\left(\frac{10^{9} \mathrm{GeV}}{m_{\chi}}\right)^{2}\left(\frac{T_{\mathrm{R}}}{10^{10} \mathrm{GeV}}\right) .
$$

\footnotetext{
${ }^{8}$ The condition of the growth of long wavelength mode in the dS era is $\nu^{2}>1 / 4$ in $(2.19)$, or $m_{\chi}^{2}<$ $(2-12 \xi) H_{\mathrm{inf}}^{2}$.
} 
Here we assumed that $\chi$ begins to oscillate before the reheating is completed: $\Gamma_{\text {inf }}<m_{\chi}$. Otherwise, the abundance is suppressed by the factor $\sim \sqrt{m_{\chi} / \Gamma_{\text {inf }}}$. This also contributes to the DM density. One crucial difference from the gravitationally produced contribution $\rho_{\chi}^{(\mathrm{GP})}$ is that the large scale fluctuation of $\chi$ has an (uncorrelated) isocurvature perturbation, which is severely constrained from observation. ${ }^{9}$ The magnitude of DM isocurvature perturbation is estimated as

$$
S_{\mathrm{DM}} \simeq R_{\chi} \frac{H_{\mathrm{inf}}}{\pi \sqrt{\left\langle\chi^{2}\right\rangle}}=R_{\chi} \sqrt{\frac{8}{3}} \frac{m_{\chi}}{H_{\mathrm{inf}}},
$$

where $R_{\chi} \equiv \rho_{\chi}^{(\mathrm{CO})} / \rho_{\mathrm{DM}}$ denotes the fraction of $\chi$ coherent oscillation energy density in the total DM energy density. The observational constraint is $S_{\mathrm{DM}} \lesssim 9 \times 10^{-6}$ [28]. Here we have focused on the range $m_{\chi} \ll H_{\mathrm{inf}}$. Otherwise, $S_{\mathrm{DM}}$ in eq. (4.3) should be replaced with $R_{\chi} \times$ $\mathcal{O}(1)$ and non-Gaussianity of the isocurvature perturbation becomes important [31, 32]. For $4 H_{\text {inf }} \gtrsim m_{\chi} \gtrsim \sqrt{2} H_{\text {inf }}$, we refer to, e.g. ref. [33] for dedicated analysis. Note that (4.1) is an asymptotic averaged value when the inflation lasts long enough and it may be possible to have larger/smaller field value in the actual Universe if $m_{\chi} \ll H_{\text {inf }}$. It is also affected by other terms like $\mathcal{L} \sim-\lambda \chi^{4}$.

Scattering of standard model particles in thermal bath also produces PGDM through the graviton exchange [11-14]. The cross section for the process like $\bar{\psi} \psi \rightarrow \chi \chi$, where $\psi$ collectively denotes the standard model fields that are in thermal bath with temperature $T$, is

$$
\sigma(\bar{\psi} \psi \rightarrow \chi \chi) \simeq \mathcal{A} \frac{T^{2}}{M_{P}^{4}},
$$

for $T \gg m_{\chi}, \mathcal{A} \simeq 1 / 24 \pi, 1 / 48 \pi$ and $1 / 12 \pi$ for complex scalar, Dirac fermion and massless vector, respectively. The final DM abundance from thermal production is dominated by those produced in the earliest epoch and estimated as

$$
\frac{\rho_{\chi}^{(\mathrm{TP})}}{s} \sim \mathcal{A} \frac{m_{\chi} T_{\mathrm{R}} T_{\mathrm{max}}^{8}}{M_{P}^{6} H_{\mathrm{inf}}^{3}} \sim \mathcal{A} \frac{m_{\chi} T_{\mathrm{R}}^{5}}{M_{P}^{4} H_{\mathrm{inf}}}
$$

where $T_{\max } \sim\left(T_{\mathrm{R}}^{2} H_{\mathrm{inf}} M_{P}\right)^{1 / 4}$ is the maximum cosmic temperature of the dilute plasma after inflation. Compared with the inflaton-induced gravitational contribution (3.20), the thermal production contribution is suppressed by $T_{\mathrm{R}}^{4} /\left(H_{\mathrm{inf}}^{2} M_{P}^{2}\right)$. Thus thermal contribution is subdominant in most cases, and some DM scenarios implementing thermal production solely were discussed in refs. [34-37], for examples. However, it is possible that $\chi$ is much heavier than the inflaton, $m_{\chi} \gg m_{\phi}$, so that all the gravitational production is not effective, while $T_{\max }>m_{\chi}$ and hence thermal production is active. In such a case, thermal production can give a dominant contribution to the PGDM abundance.

In figure 3, we show the total contribution for PGDM's relic abundance $\Omega_{\chi}$ as functions of $H_{\text {inf }}$ and DM mass $m_{\chi}$. We have chosen the reheating temperature $T_{\mathrm{R}}=10^{9} \mathrm{GeV}$

\footnotetext{
${ }^{9}$ Note on terminology: coherent oscillation may also be regarded as gravitational production, since (4.1) is induced gravitationally. We just call such long wavelength (superhorizon modes) contribution as coherent oscillation and short wavelength (subhorizon modes) one as gravitational production.
} 

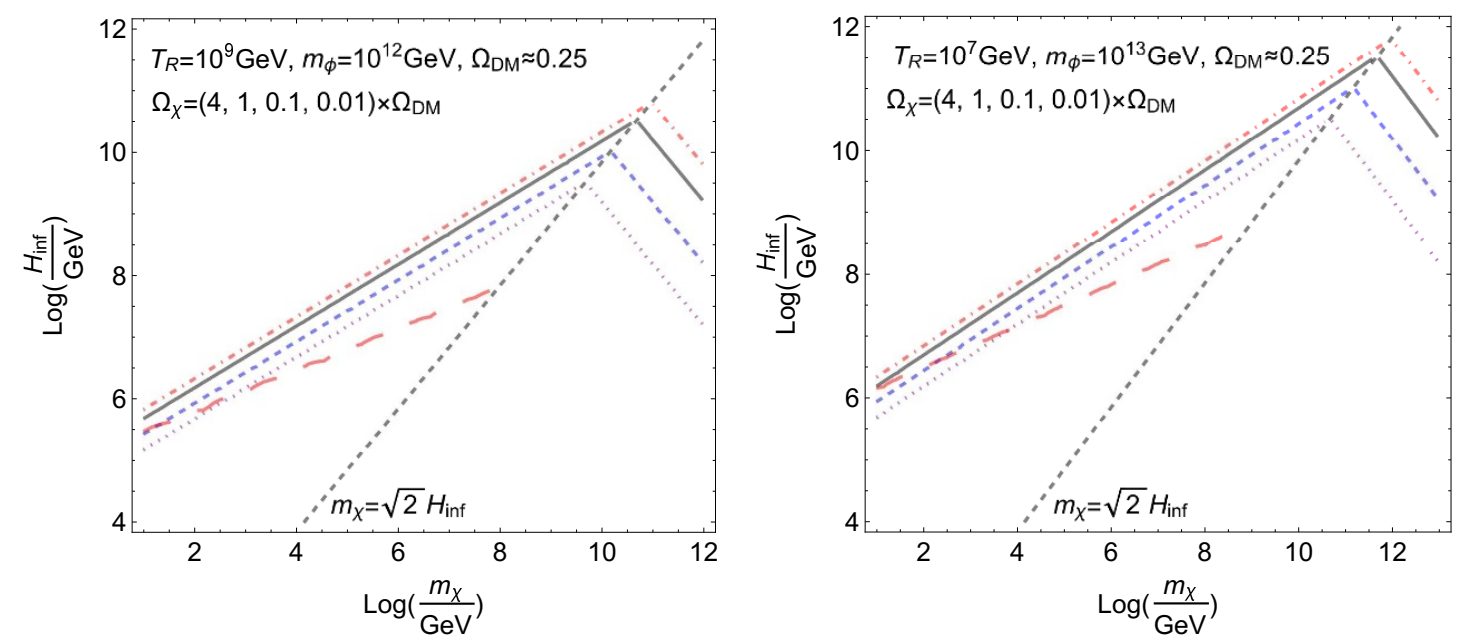

Figure 3. Illustration with reheating temperature $T_{\mathrm{R}}=10^{9} \mathrm{GeV}\left(10^{7} \mathrm{GeV}\right)$ and inflaton mass $m_{\phi}=10^{12} \mathrm{GeV}\left(10^{13} \mathrm{GeV}\right)$ for minimal coupling $\xi=0$ in the left (right) panel. Four different lines (dot-dashed, solid, dashed, and dotted) correspond to $\Omega_{\chi}=(4,1,0.1,0.01) \times \Omega_{\mathrm{DM}}$. The long dashed red line shows the isocurvature perturbation limit and region above this line is excluded. The dominant production mechanism is coherent oscillation for $m_{\chi}<\sqrt{2} H_{\text {inf }}$ and gravitational production for $m_{\chi}>\sqrt{2} H_{\mathrm{inf}}$.

$\left(10^{7} \mathrm{GeV}\right)$ and inflaton mass $m_{\phi}=10^{12} \mathrm{GeV}\left(10^{13} \mathrm{GeV}\right)$ for minimal coupling $\xi=0$ in the left (right) panel. From top to down, different lines (dot-dashed, solid, dashed, and dotted) correspond to $\Omega_{\chi}=(4,1,0.1,0.01) \times \Omega_{\mathrm{DM}}$. The long dashed line marks the isocurvature perturbation limit (4.3) and region above this line is excluded. Note that the dominant production mechanism is coherent oscillation for $m_{\chi}<\sqrt{2} H_{\text {inf }}$ and gravitational production for $m_{\chi}>\sqrt{2} H_{\text {inf }}$. In the shown parameter space, the thermal production can always be neglected. DM in the shown region might be probed by cosmic ray and CMB experiments if there is (small) $Z_{2}$ breaking term and it decays, see refs. [12,38]. Note that the constraint from isocurvature perturbation could extent to the range $m_{\chi} \lesssim 4 H_{\text {inf }}$, which indicates that the transition region close to the near-diagonal dashed line is also disfavored.

Finally we briefly comment on the case of large nonminimal coupling $\xi$. Compared with the case of minimal coupling $\xi=0$, the PGDM abundance is expected to scale as $(\xi-1 / 6)^{2}$. However, for very large $\xi \gtrsim m_{\phi}^{2} / H_{\mathrm{inf}}^{2}$, the $\chi$ field feels large tachyonic mass because the Ricci scalar is oscillating between positive and negative value during inflaton oscillation and low momentum modes are exponentially enhanced due to the tachyonic instability [8-10]. In such a case the final PGDM abundance may be greatly enhanced.

\section{Acknowledgments}

The work of Y.E. was supported in part by JSPS Research Fellowships for Young Scientists. This work was supported by the Grant-in-Aid for Scientific Research C (No.18K03609 $[\mathrm{KN}])$, Scientific Research A (No.26247042 [KN]), Young Scientists B (No.26800121 [KN]) and Innovative Areas (No. 26104009 [KN], No. 15H05888 [KN], No. 17H06359 [KN], No. $16 \mathrm{H} 06490[\mathrm{YT}])$. 


\section{A Gravitational particle production in a toy expansion model with con- formal coupling}

Here we calculate the gravitational particle production caused purely by the Hubble expansion of the Universe, i.e., without the effect of inflaton oscillation, to check the estimation of section 3.1.1. In order to get rid of the effect of inflaton oscillation, let us consider the toy model in which the $\phi$ energy density goes as

$$
\rho_{\phi}(\tau)=\frac{\rho_{\phi, i}}{1+\left(a(\tau) / a_{\text {end }}\right)^{3}} .
$$

Inflation happens for $a(\tau) \ll a_{\text {end }}$ and it smoothly connects to the MD Universe for $a(\tau) \gg$ $a_{\text {end }}$ and $\rho_{\phi, i}=3 H_{\text {inf }}^{2} M_{P}^{2}$.

Let us consider the integral (2.25) in this simplified model with the conformal coupling $\xi=1 / 6$. A general rule is that, since $\omega_{k}^{\prime} / \omega_{k}$ is a smooth function with its typical time scale at most $\sim \mathcal{H}^{-1} \sim \tau$, if the time scale of the phase factor in the integrand is much faster, the integral is exponentially suppressed as $\sim e^{-k \tau}$. Actually $\omega_{k}^{\prime} / \omega_{k}$ changes its slope at $t \simeq t_{k}$ and $t \simeq t_{\text {end }}$ and the whole integral is dominated around these epochs. First let us consider the low momentum mode $k<a_{\text {end }} m_{\chi}$. In this case we have $t_{k}<t_{\text {end }}$ and contributions around $t \simeq t_{k}$ and $t \simeq t_{\text {end }}$ are comparable. As a result, $f_{\chi}(k)=\left|\beta_{k}\right|^{2}$ is given as

$$
f_{\chi}(k) \sim \exp \left(-\frac{c m_{\chi}}{H_{\text {inf }}}\right),
$$

where we have introduced a numerical factor $c$. We will see that $c \sim 5$ fits the numerical results well. Next let us consider the high momentum mode $k>a_{\text {end }} m_{\chi}$. In this case we have $t_{k}>t_{\text {end }}$ and we obtain

$$
f_{\chi}(k) \sim \frac{a_{\text {end }}^{4} m_{\chi}^{4}}{k^{4}} \exp \left(-\frac{c k}{a_{\text {end }} H_{\text {inf }}}\right)+\exp \left[-c\left(\frac{k}{k_{c}}\right)^{3 / 2}\right],
$$

where the first (second) term corresponds to the integral around $t \sim t_{\text {end }}\left(t \sim t_{k}\right)$, and $k_{c} \equiv$ $a_{\text {end }} H_{\text {inf }}\left(m_{\chi} / H_{\text {inf }}\right)^{1 / 3}$ is defined by the momentum so that $m_{\chi} t_{k}=1$. If $m_{\chi}<H_{\text {inf }}$, the second term mostly dominates. We have omitted the mixing term since it is subdominant in most cases. Intuitively, modes with $k>k_{c}$ are always adiabatic against the expansion of the Universe. If $m_{\chi}>H_{\text {inf }}$, the first term dominates and all the modes are suppressed by at least the factor $\sim e^{-m_{\chi} / H_{\mathrm{inf}}}$, since they are all adiabatic throughout the whole history of the Universe and no significant excitations are expected. As a result, the number density due to the gravitational production in this case is approximated as

$$
n_{\chi}(\tau) \sim \mathcal{A}\left(\frac{a_{\mathrm{end}}}{a(\tau)}\right)^{3} \times\left\{\begin{array}{lll}
m_{\chi} H_{\mathrm{inf}}^{2} & \text { for } & m_{\chi} \lesssim H_{\mathrm{inf}} \\
e^{-c m_{\chi} / H_{\mathrm{inf}}} m_{\chi}^{3} & \text { for } & m_{\chi} \gtrsim H_{\mathrm{inf}}
\end{array},\right.
$$

where we find that the numerical factor $\mathcal{A}$ is weakly dependent on $m_{\chi}$ and $\mathcal{A} \sim 6 \times 10^{-4}$ for $m_{\chi} \lesssim H_{\text {inf }}$.

We solved (2.16) with the Friedmann equation $H^{2}=\rho_{\phi} /\left(3 M_{P}^{2}\right)$ by using the toy model (A.1) for the conformal coupling $\xi=1 / 6$ to estimate the gravitational particle production rate. The result of $f_{\chi}(k)$ is shown in figure 4 for $m_{\chi}=(1,0.1,0.01) \times H_{\text {inf }}$. The results are well fitted by the formula (A.2) and (A.3) with $c=5$. 


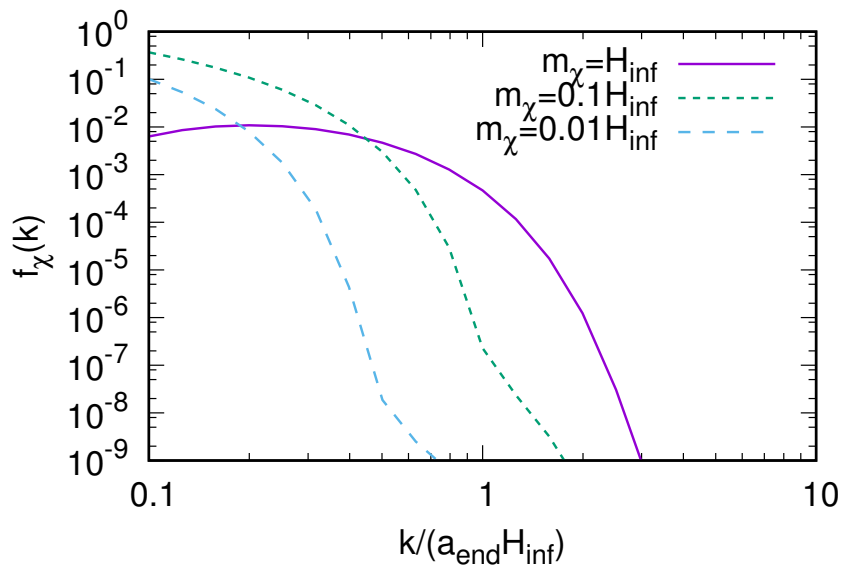

Figure 4. The phase space distribution of $\chi$ after the gravitational particle production in a toy model (A.1) with conformal coupling $\xi=1 / 6$, for $m_{\chi}=(1,0.1,0.01) \times H_{\text {inf }}$.

Open Access. This article is distributed under the terms of the Creative Commons Attribution License (CC-BY 4.0), which permits any use, distribution and reproduction in any medium, provided the original author(s) and source are credited.

\section{References}

[1] L. Parker, Quantized fields and particle creation in expanding universes. 1., Phys. Rev. 183 (1969) 1057 [INSPIRE].

[2] N.D. Birrell and P.C.W. Davies, Quantum Fields in Curved Space, Cambridge Monographs on Mathematical Physics, Cambridge University Press, Cambridge U.K. (1984).

[3] L.H. Ford, Gravitational Particle Creation and Inflation, Phys. Rev. D 35 (1987) 2955 [INSPIRE].

[4] D.J.H. Chung, E.W. Kolb and A. Riotto, Superheavy dark matter, Phys. Rev. D 59 (1999) 023501 [hep-ph/9802238] [INSPIRE].

[5] D.J.H. Chung, P. Crotty, E.W. Kolb and A. Riotto, On the Gravitational Production of Superheavy Dark Matter, Phys. Rev. D 64 (2001) 043503 [hep-ph/0104100] [InSPIRE].

[6] Y. Ema, R. Jinno, K. Mukaida and K. Nakayama, Gravitational Effects on Inflaton Decay, JCAP 05 (2015) 038 [arXiv: 1502.02475] [INSPIRE].

[7] Y. Ema, R. Jinno, K. Mukaida and K. Nakayama, Gravitational particle production in oscillating backgrounds and its cosmological implications, Phys. Rev. D 94 (2016) 063517 [arXiv: 1604.08898] [INSPIRE].

[8] B.A. Bassett and S. Liberati, Geometric reheating after inflation, Phys. Rev. D 58 (1998) 021302 [Erratum ibid. D 60 (1999) 049902] [hep-ph/9709417] [INSPIRE].

[9] S. Tsujikawa, K.-i. Maeda and T. Torii, Resonant particle production with nonminimally coupled scalar fields in preheating after inflation, Phys. Rev. D 60 (1999) 063515 [hep-ph/9901306] [INSPIRE].

[10] T. Markkanen and S. Nurmi, Dark matter from gravitational particle production at reheating, JCAP 02 (2017) 008 [arXiv: 1512.07288] [INSPIRE]. 
[11] M. Garny, M. Sandora and M.S. Sloth, Planckian Interacting Massive Particles as Dark Matter, Phys. Rev. Lett. 116 (2016) 101302 [arXiv:1511.03278] [InSPIRE].

[12] Y. Tang and Y.-L. Wu, Pure Gravitational Dark Matter, Its Mass and Signatures, Phys. Lett. B 758 (2016) 402 [arXiv:1604.04701] [INSPIRE].

[13] Y. Tang and Y.-L. Wu, On Thermal Gravitational Contribution to Particle Production and Dark Matter, Phys. Lett. B 774 (2017) 676 [arXiv:1708.05138] [InSPIRE].

[14] M. Garny, A. Palessandro, M. Sandora and M.S. Sloth, Theory and Phenomenology of Planckian Interacting Massive Particles as Dark Matter, JCAP 02 (2018) 027 [arXiv: 1709.09688] [INSPIRE].

[15] A.D. Dolgov and D.P. Kirilova, On particle creation by a time dependent scalar field, Sov. J. Nucl. Phys. 51 (1990) 172 [Yad. Fiz. 51 (1990) 273] [inSPIRE].

[16] J.H. Traschen and R.H. Brandenberger, Particle Production During Out-of-equilibrium Phase Transitions, Phys. Rev. D 42 (1990) 2491 [INSPIRE].

[17] Y. Shtanov, J.H. Traschen and R.H. Brandenberger, Universe reheating after inflation, Phys. Rev. D 51 (1995) 5438 [hep-ph/9407247] [InSPIRE].

[18] L. Kofman, A.D. Linde and A.A. Starobinsky, Towards the theory of reheating after inflation, Phys. Rev. D 56 (1997) 3258 [hep-ph/9704452] [INSPIRE].

[19] A.D. Linde, A New Inflationary Universe Scenario: A Possible Solution of the Horizon, Flatness, Homogeneity, Isotropy and Primordial Monopole Problems, Phys. Lett. B 108 (1982) 389 .

[20] A. Albrecht and P.J. Steinhardt, Cosmology for Grand Unified Theories with Radiatively Induced Symmetry Breaking, Phys. Rev. Lett. 48 (1982) 1220 [InSPIRE].

[21] K. Kumekawa, T. Moroi and T. Yanagida, Flat potential for inflaton with a discrete $R$ invariance in supergravity, Prog. Theor. Phys. 92 (1994) 437 [hep-ph/9405337] [InSPIRE].

[22] K.I. Izawa and T. Yanagida, Natural new inflation in broken supergravity, Phys. Lett. B 393 (1997) 331 [hep-ph/9608359] [inSPIRE].

[23] T. Asaka, K. Hamaguchi, M. Kawasaki and T. Yanagida, Leptogenesis in inflationary universe, Phys. Rev. D 61 (2000) 083512 [hep-ph/9907559] [INSPIRE].

[24] V.N. Senoguz and Q. Shafi, New inflation, preinflation and leptogenesis, Phys. Lett. B 596 (2004) 8 [hep-ph/0403294] [INSPIRE].

[25] L. Boubekeur and D.H. Lyth, Hilltop inflation, JCAP 07 (2005) 010 [hep-ph/0502047] [INSPIRE].

[26] Y. Ema, K. Mukaida and K. Nakayama, Electroweak Vacuum Metastability and Low-scale Inflation, JCAP 12 (2017) 030 [arXiv:1706.08920] [INSPIRE].

[27] P. Brax, J.-F. Dufaux and S. Mariadassou, Preheating after Small-Field Inflation, Phys. Rev. D 83 (2011) 103510 [arXiv:1012.4656] [INSPIRE].

[28] Planck collaboration, P.A.R. Ade et al., Planck 2015 results. XX. Constraints on inflation, Astron. Astrophys. 594 (2016) A20 [arXiv:1502.02114] [INSPIRE].

[29] A.D. Linde, Particle physics and inflationary cosmology, Contemp. Concepts Phys. 5 (1990) 1 [hep-th/0503203] [INSPIRE]. 
[30] A.D. Linde and V. Mukhanov, The curvaton web, JCAP 04 (2006) 009 [astro-ph/0511736] [INSPIRE].

[31] M. Kawasaki, K. Nakayama, T. Sekiguchi, T. Suyama and F. Takahashi, Non-Gaussianity from isocurvature perturbations, JCAP 11 (2008) 019 [arXiv:0808.0009] [INSPIRE].

[32] D. Langlois, F. Vernizzi and D. Wands, Non-linear isocurvature perturbations and non-Gaussianities, JCAP 12 (2008) 004 [arXiv:0809.4646] [INSPIRE].

[33] D.J.H. Chung and H. Yoo, Isocurvature Perturbations and Non-Gaussianity of Gravitationally Produced Nonthermal Dark Matter, Phys. Rev. D 87 (2013) 023516 [arXiv: 1110.5931] [INSPIRE].

[34] E. Babichev et al., Heavy spin-2 Dark Matter, JCAP 09 (2016) 016 [arXiv:1607.03497] [INSPIRE].

[35] E.W. Kolb and A.J. Long, Superheavy dark matter through Higgs portal operators, Phys. Rev. D 96 (2017) 103540 [arXiv:1708.04293] [INSPIRE].

[36] S.-L. Chen and Z. Kang, On UltraViolet Freeze-in Dark Matter during Reheating, JCAP 05 (2018) 036 [arXiv:1711.02556] [INSPIRE].

[37] N. Bernal, M. Dutra, Y. Mambrini, K. Olive, M. Peloso and M. Pierre, Spin-2 Portal Dark Matter, Phys. Rev. D 97 (2018) 115020 [arXiv:1803.01866] [INSPIRE].

[38] A. Ibarra, D. Tran and C. Weniger, Indirect Searches for Decaying Dark Matter, Int. J. Mod. Phys. A 28 (2013) 1330040 [arXiv:1307.6434] [INSPIRE]. 